\title{
Tuning the Energetic Landscape of Ruddlesden-Popper Perovskite Films for Efficient Solar Cells
}

Shuyan Shao, ${ }^{*}, \dagger$ Herman Duim, ${ }^{\dagger}$ Qingqian Wang, ${ }^{\ddagger}$ Bowei Xu, ${ }^{\| \odot}$ Jingin Dong, ${ }^{\dagger}$ Sampson Adjokatse, $^{\dagger}$ Graeme R. Blake, ${ }^{\dagger}$ Loredana Protesescu, ${ }^{\dagger}$ Giuseppe Portale, ${ }^{\dagger}$ (†) Jianhui Hou, ${ }^{\circ}$ Michele Saba, ${ }^{\ddagger}$ and Maria Antonietta Loi* ${ }^{\dagger}$ (i)

${ }^{\dagger}$ Zernike Institute for Advanced Materials, University of Groningen, Nijenborgh 4, 9747 AG Groningen, The Netherlands *Department of Physics, University of Cagliari, I-09042 Monserrato, Italy

"State Key Laboratory of Polymer Physics and Chemistry, Beijing National Laboratory for Molecular Sciences, Institute of Chemistry, Chinese Academy of Sciences, Beijing 100190, China

Supporting Information

ABSTRACT: Ruddlesden-Popper perovskite films deposited with different methods show very diverse phase segregation and composition. When DMSO is used as solvent, the conventional method based on spin-coating and annealing produces very poor devices, whereas the vacuum-assisted method proposed here allows obtaining devices with efficiency up to $14.14 \%$. The conventional method gives rise to a three-dimensional (3D)-like phase on the top of the film but dominant $n=2$ phase with large domains $(\sim 40 \mu \mathrm{m})$ at the bottom of the film. These $n=2$ domains are oriented with their inorganic slabs parallel to the substrate and inhibit the charge transport in the vertical direction. Consequently, severe monomolecular and bimolecular charge recombination occurs in the solar cells. Conversely, the vacuum-assisted method yields films with a 3D-

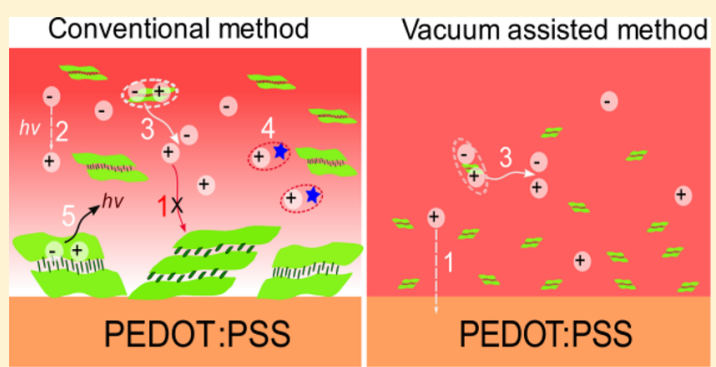

3D-like $n=2 \quad$ trap states 1 hole transport 2: bimolecular recombination 3 : energy transfer from $n=2$ to $3 D$ 4 : charge traping 5 : exciton recombination in the $n=2$ phase like phase dominant throughout their entire thickness and only a small amount of $n \leq 2$ domains of limited dimensions $(\sim 3 \mu \mathrm{m})$ at the bottom, which facilitate charge transport and reduce charge recombination.

$\mathrm{I}$ $\mathrm{n}$ the past several years, Ruddlesden-Popper perovskites (RPP) have been intensively used as light absorbers in solar cells (SCs) because of their tunable optoelectronic properties, good solution processability, and excellent ambient stability. ${ }^{1-3}$ The RPP-type perovskites have a generic formula of $\mathrm{A}_{2}{ }_{2} \mathrm{~A}_{n-1} \mathrm{~B}_{n} \mathrm{X}_{3 n+1}$, where $\mathrm{A}^{\prime}$ is a bulky aromatic or aliphatic alkylammonium cation, $A$ a small organic cation such as methylammonium (MA) or formamidinium (FA), B a divalent metal cation such as lead $(\mathrm{Pb})$ or tin $(\mathrm{Sn}), \mathrm{X}$ a halide anion $\left(\mathrm{Cl}^{-}, \mathrm{Br}^{-}\right.$, or $\left.\mathrm{I}^{-}\right)$, and $n$ the number of layers of inorganic octahedra separated by a bilayer of $\mathrm{A}^{\prime}$ cations. $^{4-8}$ When the molar ratio between $\mathrm{A}^{\prime}$ and $\mathrm{A}$ cations is adjusted, $n$ can span values from 1 (purely two-dimensaionl (2D)) to infinity (purely three-dimensional (3D)).

The $2 \mathrm{D}$ perovskites with small $n$ form natural quantum wells because of the dimensional quantum confinement and dielectric mismatch between the inorganic and organic components in the perovskite structure, where the semiconducting inorganic octahedral layers act as the potential "wells" and the organic insulating $\mathrm{A}^{\prime}$ cations as the potential "barrier".,10 As a result of this quantum and dielectric confinement, lower $n$ leads to materials of wider band gaps where the photoexcitations are excitons of high binding energy. In the case of $n=1$ or $n=2$, the $2 \mathrm{D}$ perovskites have band gaps above $2 \mathrm{eV}$ and stable excitons with large binding energy at room temperature. ${ }^{1}$ In contrast, the higher- $n$ members $(n=$ 3,4 , or 5) combine the advantages of good ambient stability of $2 \mathrm{D}$ perovskites with the broad absorption and good charge transport properties of the $3 \mathrm{D}$ perovskites and have recently become intensively used as light absorbers in solar cells. ${ }^{2,3,11}$

Because the insulating, bulky cations hinder charge transport, it is critical to grow highly oriented RPP films with the inorganic layers perpendicular to the substrate in order to guarantee efficient charge transport and collection in solar cells. To date, various strategies such as hot-casting, cesium

Received: November 3, 2019

Accepted: November 14, 2019

Published: November 14, 2019 
doping, and solvent engineering have been employed to grow RPP films with inorganic layers oriented vertically to the substrate. ${ }^{3,11,12}$ The deposition methods used for RPP films include hot-casting and room-temperature coating (conventional method). The hot-casting method, in which the substrates are preheated to a high temperature $\left(\geq 100{ }^{\circ} \mathrm{C}\right)$, leads to extremely fast solvent evaporation. ${ }^{3}$ In the case of the conventional method, substrates are kept at room temperature during the film deposition process, and thermal annealing (TA) is always performed immediately after spin-coating. While $\mathrm{Li}$ et al. reported a vacuum flash-assisted method, in which a high vacuum of $20 \mathrm{~Pa}$ was used to quickly remove the solvent in $10 \mathrm{~s}$ to grow 3D perovskite films with improved morphology, ${ }^{13}$ the vacuum-assisted method presented here has not yet been employed to grow RPP films.

Although previous works have documented the multiple perovskite phases of RPP films, they have rarely characterized segregated grains and domains of specific perovskite phases. ${ }^{11,14-16}$ Very recently, Huang et al. used high-resolution transmission electron microscopy (HRTEM) to observe coexisting $2 \mathrm{D}(n=1)$ and $3 \mathrm{D}$-like domains in RPP films deposited by the hot-casting method. ${ }^{17}$ However, the assignment of precise values of $n$ to all the perovskite phase domains in RPP films has thus far not been done. In particular, segregated domains composed of higher- $n$ structures are often confused with the 3D-like phase. A full picture to describe how the phase segregation, distribution, and composition influence the charge transport and recombination process in solar cells is thus still missing. Moreover, the way in which the processing method influences the domain size of the various phases has also not been well addressed. It is crucial to address these open questions in order to gain a full understanding of the relationship between processing techniques and solar cell performance of RPPSCs.

Herein, we investigate the phase distribution, segregation, and composition of RPP films grown by different methods and their influence on the charge recombination process in RPPSCs. When DMSO is used as the solvent, the conventional method produces very poor device performance, while the vacuum-assisted method overcomes this limitation. Films grown by the conventional method have large segregated domains and many cracks due to fast crystallization. The 3Dlike phase dominates the top of film, while the $n=2$ phase (domain size $\approx 40 \mu \mathrm{m}$ ), which coexists with a much smaller fraction of $n \geq 3$ and $n=1$ phases, dominates the bottom of the film. On the one hand, the large and parallel oriented $n=2$ domains inhibit the vertical charge transport in the solar cells. On the other hand, the excitons generated in the center of the $n=2$ domains have a higher chance to recombine. Consequently, significant monomolecular and bimolecular charge recombination occurs in the corresponding RPPSCs, leading to low device performances (PCE of $3.65 \%$ ). In contrast, perovskite films grown by the vacuum-assisted method exhibit much smaller segregated domains and compact morphology because of the slower crystallization rate. Moreover, the 3D-like phase dominates throughout the entire film, and only a small amount of $n=2$ phase (domain size $\approx 3$ $\mu \mathrm{m})$ is present at the bottom of the film. This favorable phase segregation facilitates charge transport and transfer to the charge extraction layers. Consequently, monomolecular and bimolecular recombination paths are significantly reduced in the RPPSCs, which exhibit hysteresis-free photovoltaic performance with PCE up to $14.14 \%$.
In this work, RPP films with a nominal formula of $\mathrm{PEA}_{2} \mathrm{MA}_{4} \mathrm{~Pb}_{5} \mathrm{I}_{15}$ (PEA is phenylethylammonium) were prepared by both conventional and vacuum-assisted methods. In the conventional method, the wet and colorless films were put on a hot plate at $100{ }^{\circ} \mathrm{C}$ immediately after spin-coating $(20 \mathrm{~s})$, and they turned dark red in $10 \mathrm{~s}$ because of fast evaporation of the solvent (Figure 1a). These films appear very rough,

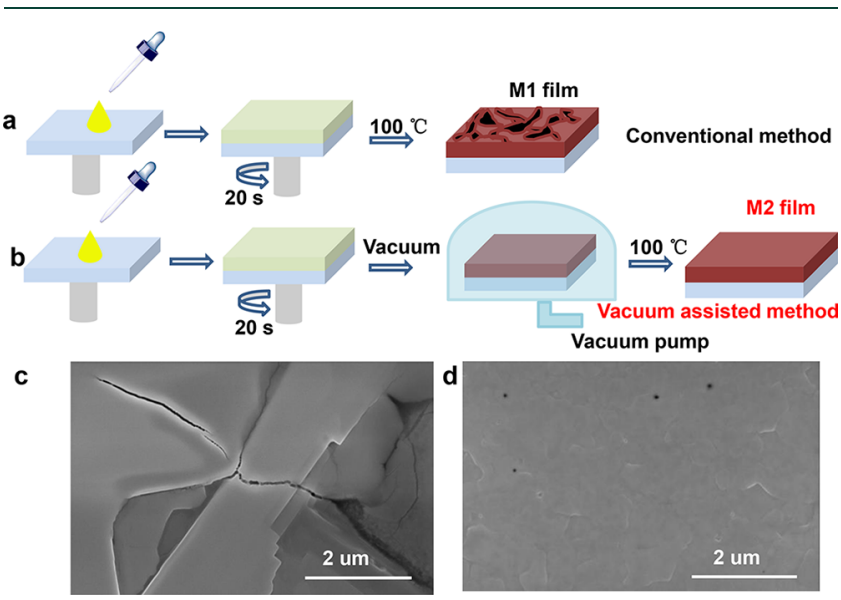

Figure 1. Preparation procedures and SEM images of the perovskite films grown by (a and c) the conventional method (M1) or (b and d) the vacuum-assisted method (M2).

nonuniform, and not very reflective to the eye. Compared to the post-thermal annealing method reported previously, a main difference is that the RPP films grown by the conventional method are still wet and composed of intermediate products before thermal annealing. ${ }^{18}$ In the vacuum-assisted method, the films were placed in vacuum immediately after the same spin-coating procedure; after $5 \mathrm{~min}$ in the chamber they appear as dark red, uniform, and very reflective (Figure $1 \mathrm{~b}$ ). The films were then removed from the vacuum chamber and put on a hot plate at $100{ }^{\circ} \mathrm{C}$ for $10 \mathrm{~min}$. The superior quality of the films grown by the vacuum-assisted method is evidenced by scanning electron microscopy (SEM) images. Films grown by the conventional method (hereafter termed M1 films) have many large cracks and pinholes (Figure 1c), creating plenty of structural defects around the open grain boundaries. ${ }^{19-24}$ In contrast, films grown by the vacuum-assisted method (hereafter termed M2 films) are compact and free of cracks, with significantly reduced density of grain boundaries (Figure 1d). This morphology is of potential benefit for suppressing trapassisted charge recombination in solar cells. ${ }^{20}$

The absorption spectra of the RPP films in Figure S1 reveal an absorption onset at $\sim 780 \mathrm{~nm}$ resembling that of $3 \mathrm{D}$ $\mathrm{MAPbI}_{3}$ films. In addition, several higher-energy peaks due to strong excitonic absorption associated with 2D phases are present, demonstrating the presence of multiple perovskite phases in both films. The M1 film exhibits pronounced excitonic absorption corresponding to the $n=2$ phase at 569 $\mathrm{nm}$ and weak exciton absorption consistent with the $n=1$ phase at $514 \mathrm{~nm}$. The M2 film displays a much weaker excitonic absorption of the $n=1$ and 2 phases, but excitonic absorption corresponding to the $n=3(609 \mathrm{~nm}), 4(640 \mathrm{~nm})$, and $5(663 \mathrm{~nm})$ phases are also observed. Such distinctive features in the absorption spectra demonstrate different phase composition in these RPP films. 

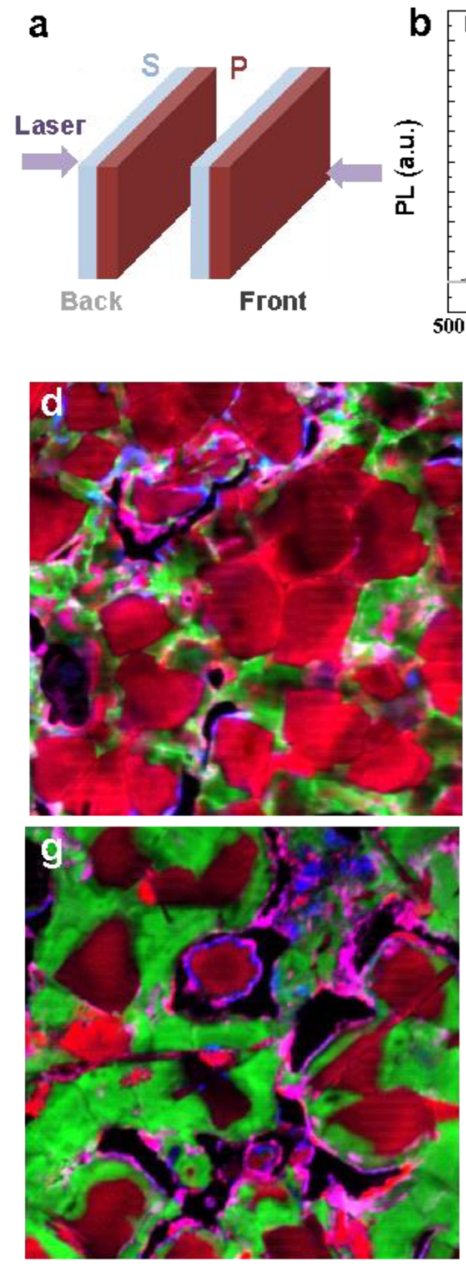
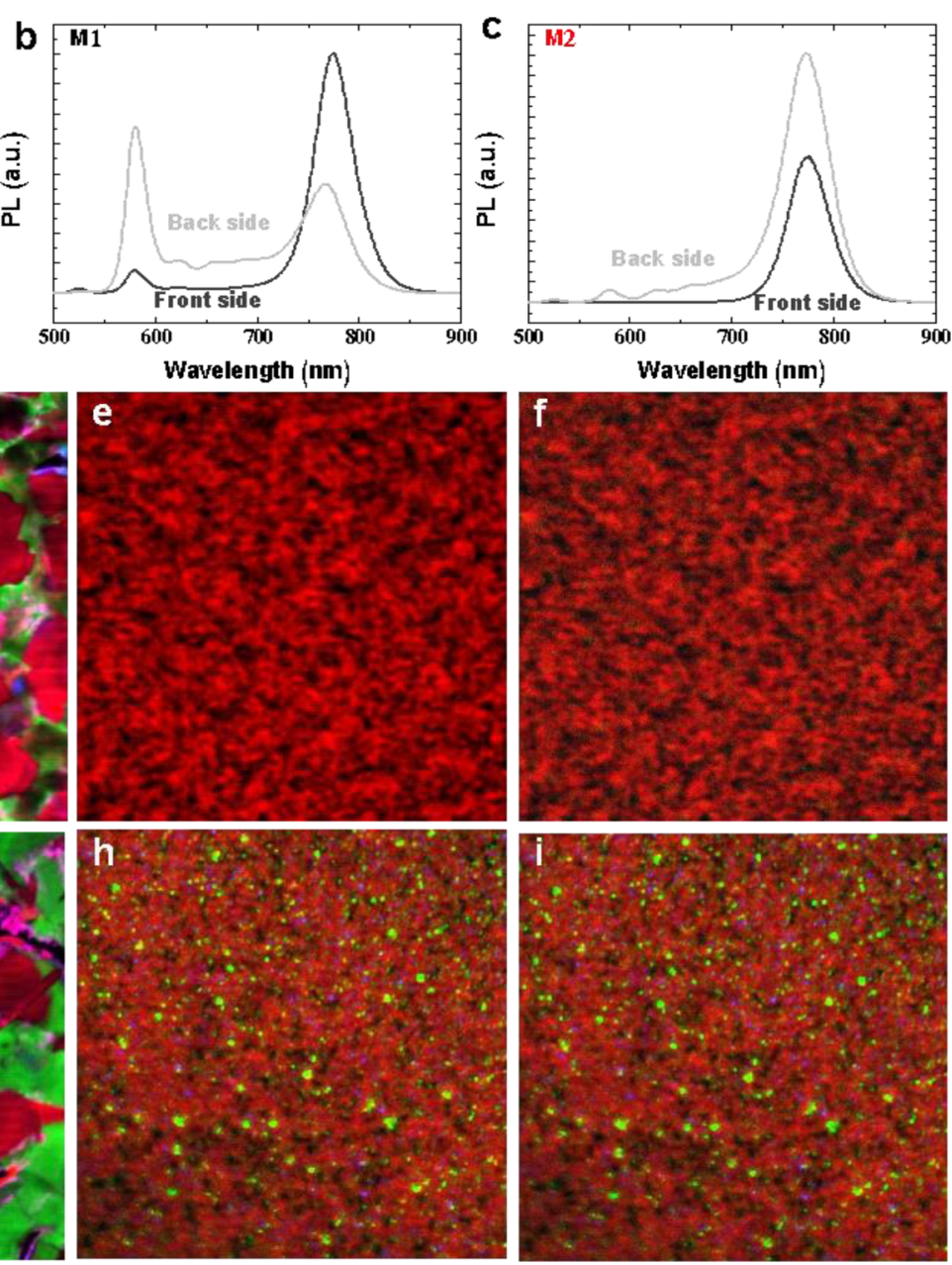

Figure 2. (a) Schematic illustration of the PL measurements, excited from the back and front sides. Steady-state PL spectra of (b) an M1 film and (c) an M2 film excited from the front (dark gray) and back (light gray). Confocal images of M1 film from (d) front side and (g) back side, and of M2 film from (e and f) front side and (h and i) back side. The films were excited by a $488 \mathrm{~nm}$ laser. The images in panels $\mathrm{d}$ and $\mathrm{g}$ were taken using four acquisition channels at wavelengths of $515 \pm 30 \mathrm{~nm}$ (blue), $590 \pm 50 \mathrm{~nm}$ (green), 650-715 nm (magenta), and 715 LP channel (red). The images in panels e and h were taken using the three channels $515 \pm 30,590 \pm 50$ and $650 \mathrm{LP}$ channel, while the micrographs in panels $\mathrm{f}$ and $\mathrm{i}$ were taken using the three channels with a $715 \mathrm{~nm}$ filter installed on the $650 \mathrm{~nm} \mathrm{LP}$ channel. Images in panels $\mathrm{d}-\mathrm{i}$ are $100 \times 100 \mu \mathrm{m}^{2}$.

Figure 2a shows the two configurations used for the steadystate and confocal laser scanning microscopy (CLSM) photoluminescence (PL) measurements. Figure $2 \mathrm{~b}$ shows the steady-state PL spectra of M1 film. Excitation from both the front and back sides give rise to PL emission typical of 3D-like phases $(\sim 775 \mathrm{~nm})$ and low-dimensional perovskite phases such as $n=1(525 \mathrm{~nm}), 2(579 \mathrm{~nm}), 3(623 \mathrm{~nm}), 4(657 \mathrm{~nm})$, and $5(682 \mathrm{~nm})$. The emission in the wavelength range from $\sim 690$ to $\sim 712 \mathrm{~nm}$ can be attributed to the $n=6$ and 7 phases. $^{25}$ To facilitate the discussion in this work, we define the $n \geq 8$ phases as $3 \mathrm{D}$-like phases, the $7 \geq n \geq 3$ as quasi-2D phase, and the $n \leq 2$ as $2 \mathrm{D}$ phase. Note that the emission at the front side is different from emission at the back side. The 3D-like perovskite emits much stronger than the lowerdimensional phases at the front side, whereas the $2 \mathrm{D}$ phases emit much stronger at the back side. These results collectively indicate a graded vertical phase distribution across the film, where $3 \mathrm{D}$-like and $2 \mathrm{D}$ phases dominate at the front and back side, respectively. It is also worth mentioning that the $n=2$ phase is dominant among the $n \leq 5$ phases, probably because of its relatively small formation energy barrier. ${ }^{26}$ Figure $2 c$ shows the PL spectra of the M2 perovskite film. The top part of this film shows exclusively emission from the 3D-like perovskite. Although emission peaks at the back side indicate the presence of multiple phases, the $\mathrm{PL}$ of the $3 \mathrm{D}$-like phase still dominates with respect to the $n \leq 7$ phases. This means that M2 films also have a graded vertical phase distribution, but they have a much smaller fraction of $n \leq 7$ phases located at the bottom.

Confocal microscopy images further confirm the presence of multiscale phase segregation domains in the films. The images composing Figure S2a,c were acquired with three different photomultiplier channels: $515 \pm 30 \mathrm{~nm}$ channel (blue color, emission assigned to the $n=1$ phase), $590 \pm 50 \mathrm{~nm}$ channel (green color, emission assigned to $n=2,3$ phases), and a 650 nm long-pass (LP) channel (red, emission assigned to $n \geq 4$ phases). In order to discern the 3D-like phase, the images in Figure S2b,d were taken using the above-mentioned channels but inserting a $715 \mathrm{~nm}$ long-pass filter to filter the emission of the $7 \geq n \geq 4$ phases in the wavelength range of $650-715 \mathrm{~nm}$. For clarity, we also created an artificial fourth detection channel (650-715 nm, marked in magenta) by performing a subtraction of two images taken with and without the $715 \mathrm{~nm}$ filter and then overlaying it on the image taken with the 715 

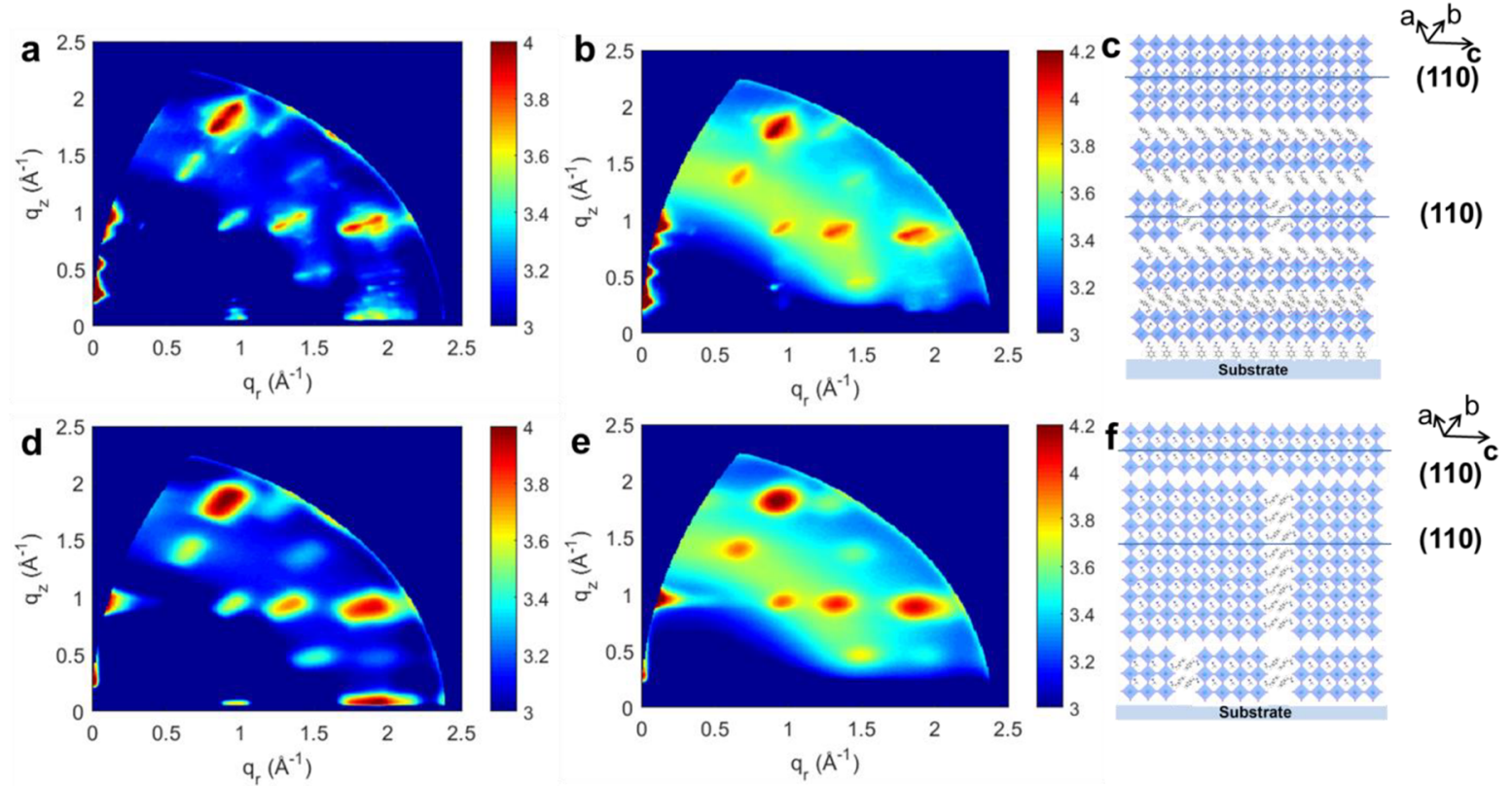

Figure 3. GIWAXS images of (a and b) M1 film and (d and e) M2 film detected at $0.25^{\circ}$ and $2.0^{\circ}$, respectively. Schematic illustration of the orientation, composition, and distribution of the perovskite phases in (c) M1 and (f) M2 films.

$\mathrm{nm}$ filter (Figure 2d,g). The front side of the M1 film shows the emission areas following the order red $>$ green $>$ magenta $\geq$ blue (Figure $2 \mathrm{~d}$ ), indicating that $3 \mathrm{D}$-like phase is more abundant than small- $n$ phases. The dominant 3D-like phase, which has segregated domains of maximum size $\sim 50 \mu \mathrm{m}$, forms an interpenetrating network surrounded by the $n \leq 7$ phases with much smaller domains. The back side of the M1 film shows emission following the order of green $>$ red $>$ magenta $>$ blue (Figure $2 \mathrm{~g}$ ). Because the quantity of the $n=3$ phase seems to be negligible compared to the $n=2$ phase, further discussion is facilitated by assuming that the large green domains correspond to the $n=2$ phase. The $n=2$ phase with segregated domains of maximum size $\sim 40 \mu \mathrm{m}$ forms an interpenetrating network and isolates the $3 \mathrm{D}$-like and the other RPP phases. The confocal images of perovskite M2 film were taken using the same three channels (with and without the 715 nm filter) as shown in Figure 2e,f,h,i. Owing to the extremely small phase segregation domains (resolution limited) of the 7 $\geq n \geq 4$ phases, we are unable to reliably create a fourth channel in this case. However, it can be clearly observed though that the front side of the M2 film shows emission only from the $3 \mathrm{D}$-like phase (domain size $\approx 2 \mu \mathrm{m}$ ), whereas the bottom surface consists of a dominant 3D-like phase, as well as a smaller proportion of $n=1$ (domain size $\approx 1 \mu \mathrm{m}$ ) and 2 (domain size $\approx 3 \mu \mathrm{m}$ ) phases.

As mentioned previously, both M1 and M2 films have a graded vertical phase distribution with the low- $n$ perovskite phases preferentially aggregating/crystallizing at the bottom. This phenomenon has also been reported by other groups in RPP films processed by either postannealing or hot-casting methods. ${ }^{17,18}$ Huang et al. attributed this vertical phase distribution to the surface crystallization mechanism. ${ }^{17}$ However, the phase and compositional segregation follow different scenarios in the two films. The M1 film contains a much larger proportion of low-dimensional perovskite phases, particularly the $n=2$ phase, than M2 films. The exact reason for this is still not clear. The molar ratios of the PEA to MA cations are $1: 1.80$ and $1: 1.53$ in the $\mathrm{M} 1$ and $\mathrm{M} 2$ films, respectively (Figure S3). These values are a bit lower than that of the precursor solution composed of stoichiometric PEAI and MAI. Thermal annealing and vacuum seem to drive some MA cations away, in agreement with previous findings by You et al. and Xie et al. ${ }^{18,27}$ We speculate that thermal annealing may provide extra driving force to enhance the crystallization of the $n=2$ phase in the conventional method, while it does not play an important role in the vacuum-assisted method because the crystallization process is complete in vacuum. The solvent evaporates much slower in the vacuum-assisted method, which enables the formation of more nucleation sites and much smaller grains for all of the perovskite phases, when compared to M1 films.

Previous studies have reported the energy transfer of the photoexcitation from the $2 \mathrm{D}$ to high- $n$ phases. ${ }^{17,18,28,29}$ In order to investigate the effect of phase segregation on the energy-transfer process, we recorded the confocal PL images of $n \geq 1$ phases under $488 \mathrm{~nm}$ excitation (Figure S4a,b) and the 3D-like phase under 488 or $632 \mathrm{~nm}$ excitation (Figure S4c-f). Interestingly, when the perovskite M1 film is excited by the $488 \mathrm{~nm}$ laser, the emission of the 3D-like phase around the grain boundaries, which are surrounded by $n \leq 3$ phases (see the regions marked by white circles for example), is significantly enhanced compared to that excited by $632 \mathrm{~nm}$ laser (Figure S4c,e). However, the emission of the central grain and of boundaries between 3D-like grains that are not surrounded by $n \leq 3$ phases remains essentially unchanged. This indicates that energy transfer from the $n \leq 3$ phases to the adjacent 3D-like phase occurs efficiently under $488 \mathrm{~nm}$ excitation. When the M1 film is excited by a $632 \mathrm{~nm}$ laser, the lack of energy transfer from $n \leq 3$ phases to the surrounding 3D-like phases leads to darker area around the grain boundaries. Recently, Herz et al. demonstrated that the excitons in the $2 \mathrm{D}$ perovskites have short diffusion length owing to the rapid recombination rate. ${ }^{15}$ In this case, the large $n=2$ domains at the bottom of the M1 film are not beneficial because the excitons have a high chance to recombine there (Figure S4c,d). Conversely, in the M2 film the small $n=2$ 
domains facilitate energy transfer between them and the 3Dlike phase.

This very large variation in the phase composition of the two perovskite films is evidenced by X-ray diffraction (XRD) patterns (Figure S5). The M1 film exhibits two intense diffraction peaks at $2 \theta=14.15^{\circ}$ and $28.48^{\circ}$, which are assigned to the (110) and (220) crystallographic planes, respectively (Figure S6). It also exhibits several evenly spaced peaks at $2 \theta=$ $3.96^{\circ}, 7.88^{\circ}, 11.82^{\circ}, 15.76^{\circ}, 19.72^{\circ}, 23.70^{\circ}$, and $27.725^{\circ}$, which are indexed as the $(00 l)$ crystallographic planes of the $n$ $=2$ phase ${ }^{4}$ In addition, two other very weak peaks at low reflection angles $\left(2 \theta=5.48^{\circ}\right.$ and $\left.10.72^{\circ}\right)$ are assigned to the (002) and (004) crystallographic planes of the $n=1$ phase. The $5 \geq n \geq 3$ phases do not show any peaks at low diffraction angle $\left(2 \theta<14.15^{\circ}\right)$, most probably because of their preferential orientation; their dominant (110) and (220) diffraction peaks are expected at similar $2 \theta$ angles to $n>5$ phases because of the very similar in-plane dimensions.

The M2 film exhibits two dominant peaks from the (110) and (220) planes, indicating preferential orientation of the perovskite domains. Diffraction peaks from the $n=1$ and 2 phases are visible but very weak because of their small volume fractions. Similar to the M1 film, the $5 \geq n \geq 3$ phases have the same preferential orientation as the $n>5$ phases, exhibiting dominant (110) and (220) planes.

In order to probe the orientation of the perovskite phases with respect to the substrate, we carried out ex-situ grazing incidence wide-angle X-ray scattering (GIWAXS) measurements. Regardless of the X-ray penetration depth, M1 and M2 films show an intense Bragg spot at $q_{z} \approx 1.0 \AA^{-1}$, whereas they show much weaker Bragg spot at $q_{y} \approx 1.0 \AA^{-1}$ (Figures 3 and S7). These results indicate that the $n \geq 3$ phases in these two films are oriented preferentially with their (110) planes parallel to the substrate and their inorganic slabs perpendicular to the substrate. ${ }^{11}$ However, M1 and M2 films show distinct features in the diffraction spots along $q_{z}$ for $q<1.0 \AA^{-1}$ due to their different phase composition. At different X-ray penetration depths, the M1 film shows intense Bragg spots of the (O0l) planes of the $n=2$ phase at $q_{z} \approx 0.29,0.58$, and $0.85 \mathrm{~A}^{-1}$, respectively (Figure $3 \mathrm{a}, \mathrm{b}$ ). In addition, M1 film shows a relatively weak Bragg spot of the (002) plane of $n=1$ phase at $q_{z} \approx 0.39 \mathrm{~A}^{-1}$. There are no observable diffraction spots of $n \leq$ 2 phases along the $q_{y}$ direction. These results indicate that the $n \leq 2$ phases of the M1 film are preferentially oriented with the inorganic layers parallel to the substrates (Figure 3c). Moreover, these results confirm the large quantity of the $n=$ 2 phase in the M1 film and are in line with the previous PL and confocal results. The M2 film shows only very weak diffraction spots of the $n=2$ phase along the $q_{z}$ direction when the X-ray fully penetrates the entire film (Figure $3 \mathrm{~d}-\mathrm{f}$ ). This proves that a very tiny amount of $n \leq 2$ phases are located at the bottom of the M2 film and are preferentially oriented with the inorganic layers parallel to the substrates (Figure $3 \mathrm{f}$ ).

Panels a and $\mathrm{b}$ in Figure 4 show the time-resolved PL decay dynamics of the perovskite films grown on glass or glass/ PEDOT:PSS substrates. At both excitation directions, the M1 film on PEDOT:PSS exhibits similar PL decay dynamics and charge carrier lifetime ( $42 \mathrm{~ns}$ at the front side and $36.5 \mathrm{~ns}$ at the back side) to that of the same film on glass ( $42.7 \mathrm{~ns}$ at the front side and $36.8 \mathrm{~ns}$ at the back side). This implies that the free charge carriers in the $3 \mathrm{D}$-like phase are not transferred to PEDOT:PSS. Figure $4 c$ depicts the decay pathways for the photoexcitations in M1 films. Except for the free charge
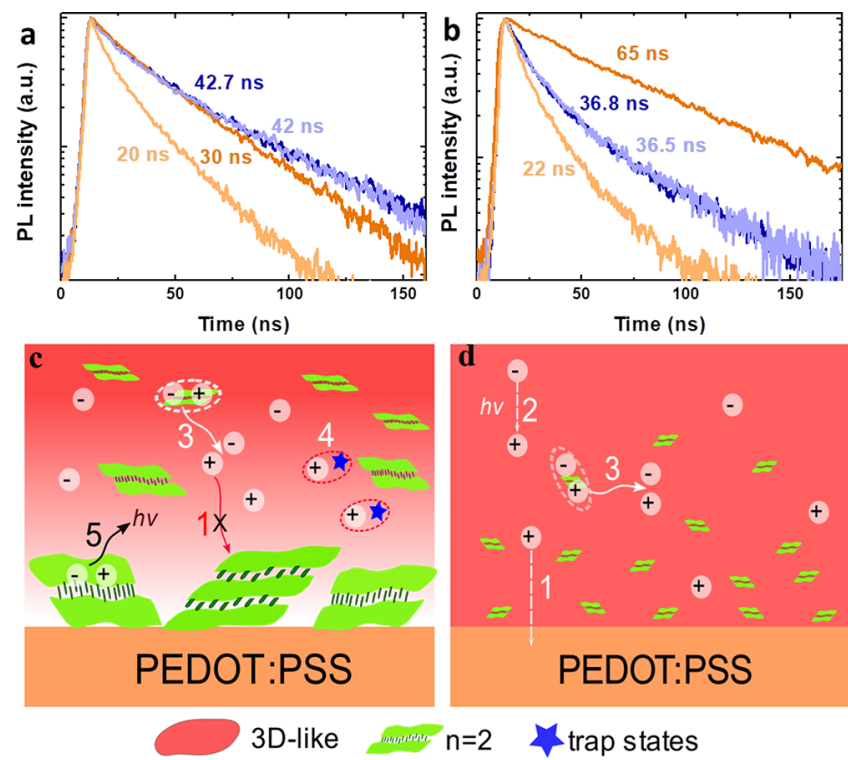

Figure 4. Time-resolved PL data recorded at $765 \mathrm{~nm}$ of M1 (blue) and M2 films (orange) on glass (darker color) and PEDOT:PSS/ glass (lighter color) substrates excited from the front side (a) and back side (b). Schematic illustration of the transfer and recombination process of the free carriers and excitons in M1 film (c) and M2 film (d). Here the red and green colors represent the 3D and $n=2$ phase, respectively. 1, hole transfer from the 3D phase to PEDOT:PSS; 2, recombination of free electrons and holes; 3, energy transfer from the $n=2$ to the 3D phase; 4 , charge trapping at the defect sites; and 5, recombination of the excitons in the $2 \mathrm{D}$ phase.

carriers at the 3D-like perovskite/PEDOT:PSS interface (process 1), those either on the top or at the bottom of the film (process 2) cannot diffuse to the PEDOT:PSS layer because of the large $n=2$ domains (Figure 4c). Consequently, they build up in the $3 \mathrm{D}$-like phase and recombine by trapassisted (process 4) and bimolecular recombination (process 2 ). The excitons in the large $n=2$ domains at the bottom of M1 film have higher chance to recombine (process 5), leading to less efficient energy transfer from low- $n$ to 3D-like phase. Moreover, the free charge carriers diffuse a shorter distance in the isolated 3D-like domains. That is why the charge carriers at the bottom of the M1 film decay faster than those on the top of the film. In contrast, M2 films on PEDOT:PSS exhibit much faster charge carrier decay (20 ns at the front side, $22.5 \mathrm{~ns}$ at the back side) compared to that on glass ( $30 \mathrm{~ns}$ at the air side, $65 \mathrm{~ns}$ at the substrate side), which indicates efficient hole transfer (process 1) from the entire perovskite film to PEDOT:PSS (Figure 4d). This process is aided by the unimpeded and barrier-free charge diffusion path formed by the vertically oriented inorganic layers, which lowers the probability of bimolecular charge recombination. At the opposite of M1 films, charge carriers at the bottom of the M2 films live much longer than those at the top surface, which obviously benefits from the energy transfer from the 2D phase to the 3D-like phase (process 3 ). More importantly, the charge carriers in M2 films survive much longer than their counterparts in M1 films. On the one hand, the charge carriers can travel longer distances in the interpenetrating 3D network of M2 films, reducing the bimolecular recombination rate. On the other hand, M2 films may have a much lower density of structural defects in absence of cracks, suppressing trap-assisted 

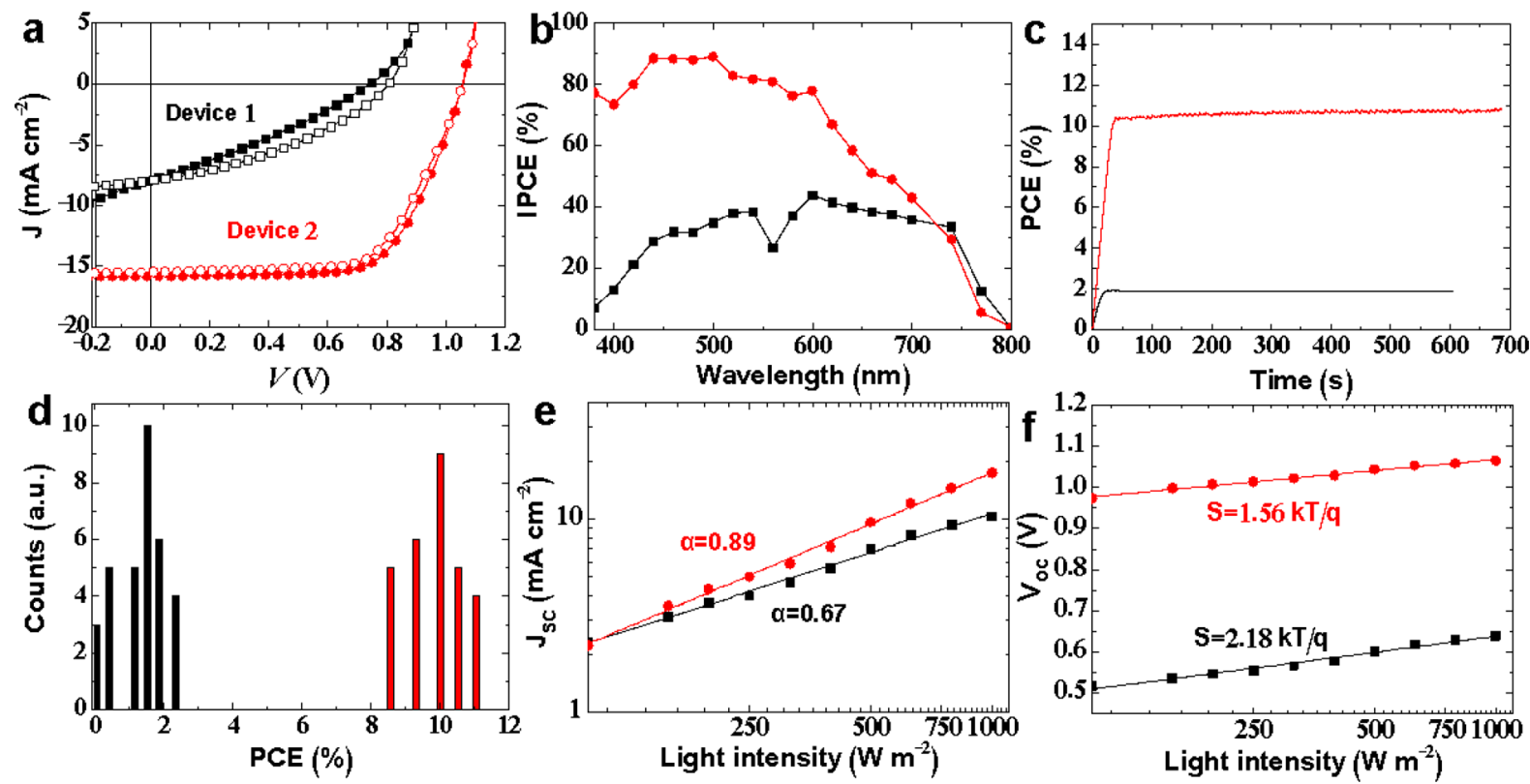

Figure 5. (a) J-V curves under one sun AM 1.5 G conditions, (b) IPCE spectra, and (c) steady-state PCE for the champion devices using M1 and M2 film as light absorber and PEDOT:PSS as HEL. (d) Statistics of the PCE; light intensity-dependent (e) $J_{\mathrm{SC}}$ and (f) $V_{\mathrm{OC}}$ of devices 1 and 2 using PEDOT:PSS as HEL.
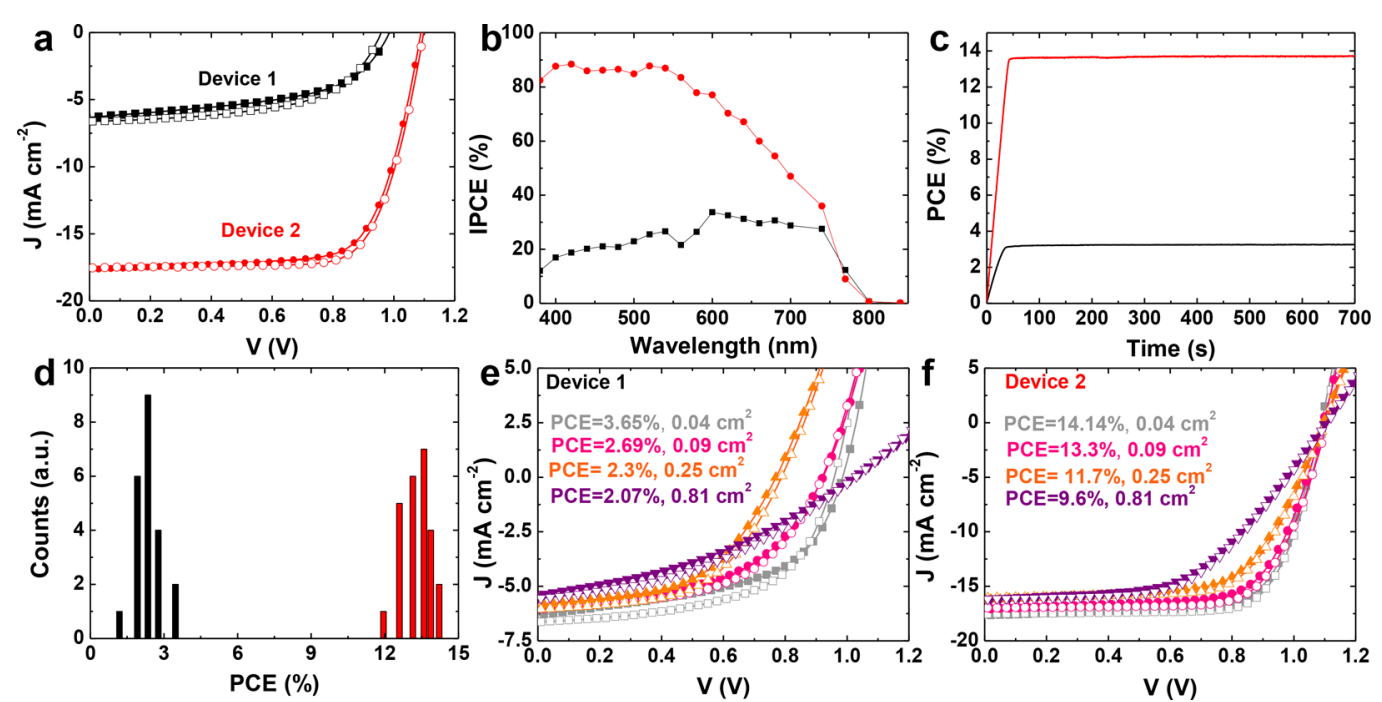

Figure 6. (a) $J-V$ curves under one sun AM 1.5 G conditions, (b) IPCE spectra, and (c) steady-state PCE for the champion devices using M1 and M2 film as light absorber and PCP-Na as HEL. (d) Statistics of the PCE over 20 devices; (e and f) $J-V$ curves under one sun AM 1.5 G conditions for devices with different cell areas using M1 and M2 films as light absorber and PCP-Na as HEL.

charge recombination rate (process 4 ). To summarize, the phase segregation domain size and composition are crucial to the charge recombination process in the perovskite films. To minimize bimolecular charge recombination, the phase segregation domains and volume fraction of the $n \leq 2$ phases should be minimized to allow the formation of an interpenetrating 3D phase network for efficient charge transport.

We now investigate how the solar cells using these perovskite films as light-harvesting layers behave. We fabricated devices with a structure comprising ITO/ PEDOT:PSS/perovskite/PCBM/ $\mathrm{C}_{60} / \mathrm{BCP} / \mathrm{Al}$ layers. The device using the $\mathrm{M} 1$ film (hereafter termed the M1 device) shows very poor performance with a $V_{\mathrm{OC}}$ of $0.81(0.74) \mathrm{V}$, a $J_{\mathrm{SC}}$ of $7.97(7.93) \mathrm{mA} \mathrm{cm}^{-2}$, a FF of $0.37(0.30)$, and a PCE of $2.40 \%$ $(1.79 \%)$ at forward (reverse) scan (Figure 5a). Note that the performance of M1 devices highly depends on the type and amount of the residue solvent in the perovskite film (immediately after spin-coating) before thermal annealing as shown in Figures S8 and S9. The device using the M2 film displays remarkable enhancement in the overall performance and has a $V_{\mathrm{OC}}$ of $1.06(1.06) \mathrm{V}$, a $J_{\mathrm{SC}}$ of $15.50(15.82) \mathrm{mA}$ $\mathrm{cm}^{-2}$, a FF of $0.65(0.66)$, and a PCE of $10.57 \%(11.07 \%)$ at forward (reverse) scan. We summarize the figures-of-merit of both type of devices in Table S1.

The conversion efficiency of the incident photon to electron (IPCE) spectra in Figure $5 \mathrm{~b}$ confirms the $J_{\mathrm{SC}}$ 's obtained from the $J-V$ measurements. Figure $5 c$ shows the steady-state PCEs of the devices tracked at the maximum power point, which are very close to those obtained from the $J-V$ measurements, further proving the reliable performance of M2 devices. To 
check the reproducibility of the HPSCs, we obtained PCE statistics over 20 devices for each deposition method (Figure $5 \mathrm{~d}$ ), which shows a consistent and clear trend of improved PCE for M2 devices.

Figure 5e shows double-logarithmic plots of light intensity (I) vs $J_{\mathrm{SC}}$ for M1 and M2 devices. Because $J_{\mathrm{SC}}$ follows a power law dependence on $I$, i.e., $J_{\mathrm{SC}} \propto I^{\alpha}$, we can obtain $\alpha$ from the slope of the $\log \left(J_{\mathrm{SC}}\right)$ versus $\log (I)$ plot. The $\log \left(J_{\mathrm{SC}}\right)$ versus $\log (I)$ plot for the M1 device shows a small slope of 0.67 due to severe bimolecular charge recombination losses. As discussed in the previous section, the free charge carriers in the high- $n$ RPP phases cannot tunnel through the potential barrier imposed by the underlying parallel-oriented $n=1$ and 2 phases to reach the electrodes. Instead, these free charges build up in the higher- $n$ RPP phases and recombine with their opposite charges, leading to significant bimolecular charge recombination. On the other hand, the large $n=2$ domains probably promote recombination of the excitons generated far from the 2D/3D interface, such that the central areas of the $n$ $=2$ phase domains do not contribute to the generation of free carriers. These factors lower the $J_{\mathrm{SC}}$ and FF of the solar cells. The corresponding plot for the M2 device shows a slope approaching unity due to the efficient charge transport. In this case, the charge transfer from the $n \leq 3$ phases to the $n>3$ phases is also facilitated by the favorable electronic coupling. The $V_{\mathrm{OC}}$ versus light intensity plot (Figure $5 \mathrm{f}$ ) of the M1 device has a large slope of approximately 2, indicating significant monomolecular charge recombination due to trapassisted or excitonic recombination, whereas the slope is much smaller for the M2 device because of the suppression of monomolecular charge recombination. Therefore, M2 devices exhibit higher $V_{\mathrm{OC}}, J_{\mathrm{SC}}$, and FF.

Finally, we investigated whether these deposition methods work in the same way when another hole transport material is used. We replaced PEDOT:PSS with a neutral conjugated polyelectrolyte PCP-Na, which has previously been shown to improve the efficiency of half lead and half tin perovskite solar cells. ${ }^{20}$ We observe a trend in device performance similar to those observed with a PEDOT:PSS as hole transport layer, as shown in Figure $6 \mathrm{a}-\mathrm{d}$. M1 devices exhibit very poor performance with a $J_{\mathrm{SC}}$ of $6.31\left(6.63 \mathrm{~mA} \mathrm{~cm}{ }^{-2}\right)$, a $V_{\mathrm{OC}}$ of $0.985(0.959) \mathrm{V}$, a FF of $0.53(0.57)$, and a PCE of $3.31 \%$ (3.65\%) at forward (reverse) sweep direction. M2 devices show significantly higher overall performance, with a high $V_{\mathrm{OC}}$ of $1.1(1.1 \mathrm{~V})$, a $J_{\mathrm{SC}}$ of $17.09(17.52) \mathrm{mA} \mathrm{cm}^{-2}$, a FF of 0.73 (0.73), and a PCE of $13.70 \%(14.14 \%)$ at the forward (reverse) scan, respectively. The reliable performance of these devices was further proven by the IPCE spectra (Figure $6 \mathrm{~b}$ ), the statistics of the PCE (Figure 6d), and the steady-state PCE (Figure 6c). Moreover, we fabricated devices with different areas as shown in Figure 6e,f. When the area is increased to $0.81 \mathrm{~cm}^{2}, \mathrm{M} 2$ devices display a PCE of $9.6 \%$, which is much higher than the corresponding value of $2.07 \%$ for M1 devices.

In conclusion, we investigated the multiscale phase and composition segregation in RPP films. Perovskite films grown by the conventional method are composed of large domains of 3D-like and 2D phases, which dominate the top and bottom part of the films, respectively. The large and parallel oriented $n$ $=2$ phase domains not only inhibit free charge carrier transport in the vertical direction but also increase the recombination of the excitons, causing severe first- and second-order charge recombination in the RPPSCs. In contrast, perovskite films grown by the vacuum-assisted method have much smaller domains. These films are composed dominantly by the 3D phase throughout the entire thickness, and only a small fraction of $n \leq 2$ phases with small domains is present at the bottom surface. This favorable phase distribution, segregation, and composition facilitates charge transport and transfer to the interfacial layers, suppressing the first- and second-order charge recombination significantly in the RPPSCs. Consequently, devices based on perovskite films grown using the vacuumassisted method have much higher device performance. Our work demonstrates that the segregation, concentration, and orientation of $n \leq 22 \mathrm{D}$ phases are critical for device performance.

\section{ASSOCIATED CONTENT}

\section{S Supporting Information}

The Supporting Information is available free of charge at https://pubs.acs.org/doi/10.1021/acsenergylett.9b02397.

Experimental details, UV-vis absorption spectra, laser scanning confocal images of different samples, XRD and GIWAX measurements, $1 \mathrm{H}-\mathrm{NMR}$ spectra, JV characteristics of devices processed with different solvent mixtures, table with figures of merit for different devices (PDF)

\section{AUTHOR INFORMATION}

\section{Corresponding Authors}

*E-mail: S.Shao@rug.nl.

*E-mail: M.A.Loi@rug.nl.

ORCID

Shuyan Shao: 0000-0001-7005-8484

Bowei Xu: 0000-0001-6467-6147

Graeme R. Blake: 0000-0001-9531-7649

Loredana Protesescu: 0000-0002-9776-9881

Giuseppe Portale: 0000-0002-4903-3159

Jianhui Hou: 0000-0002-2105-6922

Michele Saba: 0000-0001-6416-3122

Maria Antonietta Loi: 0000-0002-7985-7431

\section{Notes}

The authors declare no competing financial interest.

\section{ACKNOWLEDGMENTS}

This work is part of the research program of the Foundation for Fundamental Research on Matter (FOM), which is part of The Netherlands Organization for Scientific Research (NWO). This is a publication of the FOM-focus Group "Next Generation Organic Photovoltaics," participating in the Dutch Institute for Fundamental Energy Research (DIFFER). B.X. acknowledges the financial support from the Natural Science Foundation of China (Grant No. 21875263). We thank P. van der Meulen and K. Kahlot for performing the NMR measurements. We thank Arjen Kamp and Teo Zaharia for their kind technical support in the laboratory.

\section{REFERENCES}

(1) Smith, I. C.; Hoke, E. T.; Solis-Ibarra, D.; McGehee, M. D.; Karunadasa, H. I. A Layered Hybrid Perovskite Solar-Cell Absorber with Enhanced Moisture Stability. Angew. Chem. 2014, 126 (42), 11414-11417.

(2) Cao, D. H.; Stoumpos, C. C.; Farha, O. K.; Hupp, J. T.; Kanatzidis, M. G. 2D Homologous Perovskites as Light-Absorbing 
Materials for Solar Cell Applications. J. Am. Chem. Soc. 2015, 137 (24), 7843-7850.

(3) Tsai, H.; Nie, W.; Blancon, J.-C.; Stoumpos, C. C.; Asadpour, R.; Harutyunyan, B.; Neukirch, A. J.; Verduzco, R.; Crochet, J. J.; Tretiak, S.; et al. High-Efficiency Two-Dimensional Ruddlesden-Popper Perovskite Solar Cells. Nature 2016, 536, 312.

(4) Stoumpos, C. C.; Cao, D. H.; Clark, D. J.; Young, J.; Rondinelli, J. M.; Jang, J. I.; Hupp, J. T.; Kanatzidis, M. G. Ruddlesden-Popper Hybrid Lead Iodide Perovskite 2D Homologous Semiconductors. Chem. Mater. 2016, 28 (8), 2852-2867.

(5) Calabrese, J.; Jones, N. L.; Harlow, R. L.; Herron, N.; Thorn, D. L.; Wang, Y. Preparation and Characterization of Layered Lead Halide Compounds. J. Am. Chem. Soc. 1991, 113 (6), 2328-2330.

(6) Ishihara, T.; Takahashi, J.; Goto, T. Exciton State in TwoDimensional Perovskite Semiconductor $\left(\mathrm{C}_{10} \mathrm{H}_{21} \mathrm{NH}_{3}\right)_{2} \mathrm{PbI}_{4}$. Solid State Commun. 1989, 69 (9), 933-936.

(7) Mitzi, D. B.; Wang, S.; Feild, C. A.; Chess, C. A.; Guloy, A. M. Conducting Layered Organic-Inorganic Halides Containing $\langle 110\rangle$ Oriented Perovskite Sheets. Science (Washington, DC, U. S.) 1995, 267 (5203), 1473-1476.

(8) Mitzi, D. B.; Feild, C. A.; Harrison, W. T. A.; Guloy, A. M. Conducting Tin Halides with a Layered Organic-Based Perovskite Structure. Nature 1994, 369 (6480), 467-469.

(9) Kamminga, M. E.; Fang, H.-H.; Filip, M. R.; Giustino, F.; Baas, J.; Blake, G. R.; Loi, M. A.; Palstra, T. T. M. Confinement Effects in Low-Dimensional Lead Iodide Perovskite Hybrids. Chem. Mater. 2016, 28 (13), 4554-4562.

(10) Katan, C.; Mercier, N.; Even, J. Quantum and Dielectric Confinement Effects in Lower-Dimensional Hybrid Perovskite Semiconductors. Chem. Rev. 2019, 119 (5), 3140-3192.

(11) Qing, J.; Liu, X.-K.; Li, M.; Liu, F.; Yuan, Z.; Tiukalova, E.; Yan, Z.; Duchamp, M.; Chen, S.; Wang, Y.; et al. Aligned and Graded Type-II Ruddlesden-Popper Perovskite Films for Efficient Solar Cells. Adv. Energy Mater. 2018, 8 (21), 1800185.

(12) Zhang, X.; Ren, X.; Liu, B.; Munir, R.; Zhu, X.; Yang, D.; Li, J.; Liu, Y.; Smilgies, D.-M.; Li, R.; et al. Stable High Efficiency TwoDimensional Perovskite Solar Cells via Cesium Doping. Energy Environ. Sci. 2017, 10 (10), 2095-2102.

(13) Li, X.; Bi, D.; Yi, C.; Décoppet, J.-D.; Luo, J.; Zakeeruddin, S. M.; Hagfeldt, A.; Grätzel, M. A Vacuum Flash-Assisted Solution Process for High-Efficiency Large-Area Perovskite Solar Cells. Science 2016, 353 (6294), 58-62.

(14) Shao, S.; Liu, J.; Portale, G.; Fang, H.-H.; Blake, G. R.; ten Brink, G. H.; Koster, L. J. A.; Loi, M. A. Highly Reproducible SnBased Hybrid Perovskite Solar Cells with 9\% Efficiency. Adv. Energy Mater. 2018, 8 (4), 1702019.

(15) Milot, R. L.; Sutton, R. J.; Eperon, G. E.; Haghighirad, A. A.; Martinez Hardigree, J.; Miranda, L.; Snaith, H. J.; Johnston, M. B.; Herz, L. M. Charge-Carrier Dynamics in 2D Hybrid Metal-Halide Perovskites. Nano Lett. 2016, 16 (11), 7001-7007.

(16) Shao, S.; Dong, J.; Duim, H.; ten Brink, G. H.; Blake, G. R.; Portale, G.; Loi, M. A. Enhancing the Crystallinity and Perfecting the Orientation of Formamidinium Tin Iodide for Highly Efficient SnBased Perovskite Solar Cells. Nano Energy 2019, 60, 810-816.

(17) Lin, Y.; Fang, Y.; Zhao, J.; Shao, Y.; Stuard, S. J.; Nahid, M. M.; Ade, H.; Wang, Q.; Shield, J. E.; Zhou, N.; et al. Unveiling the Operation Mechanism of Layered Perovskite Solar Cells. Nat. Commun. 2019, 10 (1), 1008.

(18) Yan, L.; Hu, J.; Guo, Z.; Chen, H.; Toney, M. F.; Moran, A. M.; You, W. General Post-Annealing Method Enables High-Efficiency Two-Dimensional Perovskite Solar Cells. ACS Appl. Mater. Interfaces 2018, 10 (39), 33187-33197.

(19) Shao, S.; Abdu-Aguye, M.; Qiu, L.; Lai, L.-H.; Liu, J.; Adjokatse, S.; Jahani, F.; Kamminga, M. E.; ten Brink, G. H.; Palstra, T. T. M.; et al. Elimination of the Light Soaking Effect and Performance Enhancement in Perovskite Solar Cells Using a Fullerene Derivative. Energy Environ. Sci. 2016, 9 (7), 2444-2452.

(20) Shao, S.; Abdu-Aguye, M.; Sherkar, T. S.; Fang, H.-H.; Adjokatse, S.; ten Brink, G.; Kooi, B. J.; Koster, L. J. A.; Loi, M. A.
The Effect of the Microstructure on Trap-Assisted Recombination and Light Soaking Phenomenon in Hybrid Perovskite Solar Cells. Adv. Funct. Mater. 2016, 26 (44), 8094-8102.

(21) Liu, J.; Lu, S.; Zhu, L.; Li, X.; Choy, W. C. H. PerovskiteOrganic Hybrid Tandem Solar Cells Using a Nanostructured Perovskite Layer as the Light Window and a PFN/Doped-MoO3/ MoO3Multilayer as the Interconnecting Layer. Nanoscale 2016, 8 (6), 3638-3646.

(22) Qian, L.; Sun, Y.; Sun, M.; Fang, Z.; Li, L.; Xie, D.; Li, C.; Ding, L. 2D Perovskite Microsheets for High-Performance Photodetectors. J. Mater. Chem. C 2019, 7 (18), 5353-5358.

(23) Shrestha, S.; Matt, G. J.; Osvet, A.; Niesner, D.; Hock, R.; Brabec, C. J. Assessing Temperature Dependence of Drift Mobility in Methylammonium Lead Iodide Perovskite Single Crystals. J. Phys. Chem. C 2018, 122 (11), 5935-5939.

(24) Shao, S.; Cui, Y.; Duim, H.; Qiu, X.; Dong, J.; ten Brink, G. H.; Portale, G.; Chiechi, R. C.; Zhang, S.; Hou, J.; et al. Enhancing the Performance of the Half Tin and Half Lead Perovskite Solar Cells by Suppression of the Bulk and Interfacial Charge Recombination. Adv. Mater. 2018, 30 (35), 1803703.

(25) Soe, C. M. M.; Nagabhushana, G. P.; Shivaramaiah, R.; Tsai, H.; Nie, W.; Blancon, J.-C.; Melkonyan, F.; Cao, D. H.; Traoré, B.; Pedesseau, L.; et al. Structural and Thermodynamic Limits of Layer Thickness in 2D Halide Perovskites. Proc. Natl. Acad. Sci. U. S. A. 2019, 116 (1), 58-66.

(26) Ma, C.; Lo, M.-F.; Lee, C.-S. A Simple Method for Phase Control in Two-Dimensional Perovskite Solar Cells. J. Mater. Chem. A 2018, 6 (39), 18871-18876.

(27) Xie, F. X.; Zhang, D.; Su, H.; Ren, X.; Wong, K. S.; Grätzel, M.; Choy, W. C. H. Vacuum-Assisted Thermal Annealing of CH3NH3PbI3 for Highly Stable and Efficient Perovskite Solar Cells. ACS Nano 2015, 9 (1), 639-646.

(28) Proppe, A. H.; Quintero-Bermudez, R.; Tan, H.; Voznyy, O.; Kelley, S. O.; Sargent, E. H. Synthetic Control over Quantum Well Width Distribution and Carrier Migration in Low-Dimensional Perovskite Photovoltaics. J. Am. Chem. Soc. 2018, 140 (8), 28902896.

(29) Shang, Q.; Wang, Y.; Zhong, Y.; Mi, Y.; Qin, L.; Zhao, Y.; Qiu, X.; Liu, X.; Zhang, Q. Unveiling Structurally Engineered Carrier Dynamics in Hybrid Quasi-Two-Dimensional Perovskite Thin Films toward Controllable Emission. J. Phys. Chem. Lett. 2017, 8 (18), 4431-4438. 